\title{
Modafinil ameliorates excessive daytime sleepiness after traumatic brain injury
}

Kaiser, P R ; Valko, P O ; Werth, E ; Thomann, J ; Meier, J ; Stocker, R ; Bassetti, C L ; Baumann, C $\mathrm{R}$

\begin{abstract}
BACKGROUND: Excessive daytime sleepiness (EDS) and fatigue are common symptoms after traumatic brain injury (TBI), but there is no specific treatment for affected patients. With this pilot study, we aimed at studying the effect of daily modafinil on posttraumatic EDS and fatigue. METHODS: We conducted a prospective, double-blind, randomized, placebo-controlled pilot study in 20 patients with TBI who had fatigue or EDS or both. After baseline examinations (questionnaires including the Epworth Sleepiness Scale to assess EDS and the Fatigue Severity Scale to assess fatigue, actigraphy, polysomnography, maintenance of wakefulness test, and psychomotor vigilance test), 10 patients received 100 to $200 \mathrm{mg}$ modafinil every morning, and 10 patients were treated with placebo. After a 6-week treatment period, all examinations were repeated. RESULTS: EDS improved significantly in patients with TBI who were treated with modafinil, compared with the placebo group. Similarly, the ability to stay awake on the maintenance of wakefulness test improved only in the modafinil group. Modafinil, however, had no impact on posttraumatic fatigue. Clinically relevant side effects were not observed. CONCLUSION: This study indicates that modafinil is effective and well tolerated in the treatment of posttraumatic EDS but not of fatigue. CLASSIFICATION OF EVIDENCE: This study provides Class I evidence that modafinil (100-200 mg daily) improves posttraumatic EDS compared with placebo. This study provides Class I evidence that modafinil (100-200 mg daily) does not improve posttraumatic fatigue compared with placebo.
\end{abstract}

DOI: https://doi.org/10.1212/WNL.0b013e3181fd62a2

Posted at the Zurich Open Repository and Archive, University of Zurich

ZORA URL: https://doi.org/10.5167/uzh-43366

Journal Article

Published Version

Originally published at:

Kaiser, P R; Valko, P O; Werth, E; Thomann, J; Meier, J; Stocker, R; Bassetti, C L; Baumann, C R (2010). Modafinil ameliorates excessive daytime sleepiness after traumatic brain injury. Neurology, 75(20):1780-1785.

DOI: https://doi.org/10.1212/WNL.0b013e3181fd62a2 
P.R. Kaiser, MSc*

P.O. Valko, $\mathrm{MD}^{*}$

E. Werth, PhD

J. Thomann, MSc

J. Meier

R. Stocker, MD

C.L. Bassetti, MD

C.R. Baumann, MD

Address correspondence and reprint requests to Dr. Christian R. Baumann, Department of Neurology, University Hospital Zurich, Frauenklinikstrasse 26, 8091 Zurich, Switzerland christian.baumann@usz.ch

\section{Modafinil ameliorates excessive daytime sleepiness after traumatic brain injury}

吅 $\underline{\underline{\underline{\underline{\underline{A}}}}}$

\section{ABSTRACT}

Background: Excessive daytime sleepiness (EDS) and fatigue are common symptoms after traumatic brain injury (TBI), but there is no specific treatment for affected patients. With this pilot study, we aimed at studying the effect of daily modafinil on posttraumatic EDS and fatigue.

Methods: We conducted a prospective, double-blind, randomized, placebo-controlled pilot study in 20 patients with TBI who had fatigue or EDS or both. After baseline examinations (questionnaires including the Epworth Sleepiness Scale to assess EDS and the Fatigue Severity Scale to assess fatigue, actigraphy, polysomnography, maintenance of wakefulness test, and psychomotor vigilance test), 10 patients received 100 to 200 mg modafinil every morning, and 10 patients were treated with placebo. After a 6-week treatment period, all examinations were repeated.

Results: EDS improved significantly in patients with TBI who were treated with modafinil, compared with the placebo group. Similarly, the ability to stay awake on the maintenance of wakefulness test improved only in the modafinil group. Modafinil, however, had no impact on posttraumatic fatigue. Clinically relevant side effects were not observed.

Conclusion: This study indicates that modafinil is effective and well tolerated in the treatment of posttraumatic EDS but not of fatigue.

Classification of evidence: This study provides Class I evidence that modafinil (100-200 mg daily) improves posttraumatic EDS compared with placebo. This study provides Class I evidence that modafinil (100-200 mg daily) does not improve posttraumatic fatigue compared with placebo. Neurology ${ }^{\circledR} 2010 ; 75: 1780-1785$

GLOSSARY

EDS = excessive daytime sleepiness; ESS = Epworth Sleepiness Scale; FSS = Fatigue Severity Scale; GCS = Glasgow Coma Scale; MWT = maintenance of wakefulness test; TBI = traumatic brain injury.

Traumatic brain injury (TBI) occurs in about 1.4 million people in the United States each year, corresponding to an incidence of 460 per 100,000 per year. ${ }^{1}$ Another study estimated that 3.17 million US citizens (more than 1\% of the total US population) have long-term neurologic disability that resulted from TBI. ${ }^{2}$ Thus, TBI causes the largest number of disability-adjusted life-years lost to death and to varying degrees of disability, including posttraumatic sleep-wake disturbances. ${ }^{3}$

Excessive daytime sleepiness (EDS) and fatigue are frequent symptoms after TBI. In our prospective study on posttraumatic sleep-wake disturbances, we studied 65 consecutive patients by means of questionnaires and sleep laboratory examinations 6 months after trauma. ${ }^{4}$ We found EDS in $38 \%$ and fatigue in $17 \%$ of our patients. Our findings are in line with other reports on posttraumatic sleep-wake disturbances. ${ }^{5-7}$ The etiology of posttraumatic EDS is not known. Recently, we found a partial loss of wake-promoting hypothalamic hypocretin (orexin) neurons after fatal TBI. ${ }^{8}$ EDS and fatigue significantly impair quality of life and daytime functioning, including professional performances and social activities. ${ }^{4}$ Despite their high inci-

\footnotetext{
*The first 2 authors contributed equally to this study.

From the Departments of Neurology (P.R.K., P.O.V., E.W., J.T., J.M., C.L.B., C.R.B.) and Surgical Intensive Care (R.S.), University Hospital Zurich; and Department of Neurology (C.L.B.), Neurocenter of Southern Switzerland, Ospedale Civico, Lugano, Switzerland.

Study funding: Supported by Schweizerischer Versicherungsverband (SVV), Zurich, Switzerland, and Cephalon GmbH, Martinsried, Germany.

Disclosure: Author disclosures are provided at the end of the article.
} 
dences, posttraumatic EDS and fatigue are still underestimated, and there is no treatment for affected patients.

Thus, with this pilot study, we aimed at studying the effect of daily modafinil on posttraumatic EDS and fatigue. Modafinil (2[(diphenylmethyl) sulfinyl] acetamide) has wake-promoting properties and is approved by the US Food and Drug Administration for the treatment of EDS in patients with narcolepsy, obstructive sleep apnea syndrome, and shift work sleep disorder.

METHODS This prospective study was conducted at the Department of Neurology of the University Hospital of Zurich, Switzerland, between January 2007 and June 2009.

Subjects. In this prospective, double-blind, randomized, placebo-controlled pilot study, we included 20 consecutive patients from our first study on posttraumatic sleep-wake disturbances. ${ }^{4}$ All patients were admitted for closed mild to severe TBI to our surgical intensive care unit, where they were recruited for this study. TBI severity was quantified with the initial Glasgow Coma Scale (GCS) examination, which was performed immediately after trauma (mild TBI: GCS 13-15, moderate TBI: GCS 9-12, severe TBI: GCS 3-8). Admission to the surgical intensive care unit was for mild to severe TBI. The mandatory inclusion criterion was the presence of fatigue or EDS or both since TBI. Patients with neurologic, psychiatric, or other disorders and medication that might cause sleep-wake disturbances were not included. Furthermore, we excluded patients who revealed significant sleep-wake disturbances other than posttraumatic vigilance impairment at baseline examinations. Patients with chronic sleep deprivation were also excluded. The latter was diagnosed in patients with EDS who slept $\geq 2$ hours more on weekends or during holidays than during weekdays. All exclusions were made before randomization.

Fatigue was assessed with the Fatigue Severity Scale (FSS), and EDS was assessed with the Epworth Sleepiness Scale (ESS). Both scales have been validated in German. ${ }^{9} 10$ Fatigue is diagnosed in patients with FSS values above 4.0, and EDS is diagnosed in those with ESS scores $\geq 10$. Before randomization, written informed consent was obtained from all patients.

Baseline examinations. At baseline, patients were administered the Beck Depression and Anxiety Inventory, a standard questionnaire to assess depression and anxiety symptoms. ${ }^{11}$ To rule out chronic sleep deprivation, patients were examined by 2 weeks of actigraphy studies. Actigraphy is a noninvasive method to monitor activity/rest cycles over a defined period of time. Sleep and physical activity levels were recorded by wrist actigraphy. The actimeter includes a light sensor and is worn on the nondominant wrist (Actiwatch, CamNtech Ltd., Cambridge, UK). ${ }^{12}$ With this software, the determination of time asleep and time awake relies on an algorithm using the activity data recorded by the Actiwatch in a series of linked calculations. During actigraphy recordings, all patients simultaneously filled in sleep logs.

Immediately after actigraphy, conventional nocturnal polysomnography was recorded from $11 \mathrm{PM}$ to $7 \mathrm{AM}$. We used a 16-channel recording system (Embla A10, Embla, Broomfield,
CO). Sleep-stage scoring was performed visually by the first authors according to standard criteria. ${ }^{13}$ Patients with significant sleep-wake disturbances, such as obstructive sleep apnea, detected during polysomnographic recordings were excluded from the study. The day after polysomnography, all patients were examined by a maintenance of wakefulness test (MWT) ${ }^{14}$ This test measures the ability to stay awake for 40 minutes while sitting quietly in a dark room and is performed every 2 hours, in total 4 times a day. Mean sleep latency in the MWT was defined as the time from lights off to the first epoch of any sleep stage. At the same day, before every MWT test, we examined the patients' vigilance with the psychomotor vigilance test. This test measures reaction times of patients to the sudden appearance of a timekeeper on a black computer screen. ${ }^{15}$ The test lasts 10 minutes and assesses the number of lapses, defined as times the subject does not respond to the signal in time, and calculates reaction times. Intake of caffeine and medication with known effects on vigilance and sleepiness were not allowed during days and nights with sleep recordings and vigilance tests.

Randomization and follow-up examinations. After completion and evaluation of all tests, included patients were randomly assigned to either the modafinil group or the placebo group. All study procedures were performed in a strict doubleblind manner. For allocation concealment, labeling of masked study medication (modafinil or placebo) by manufacturing of numbered containers was performed centrally by the Pharmacy of the University Hospital of Zurich. All patients in the treatment group started with 1 oral capsule (corresponding to 100 $\mathrm{mg}$ modafinil per day in the treatment group), administered in the morning. Every 2 weeks, a phone call from the first author evaluated the subjective response to treatment, including efficacy and side effects. In patients without clear efficacy and without side effects, the dose was doubled: 1 oral capsule in the morning and 1 at noon. In patients with troublesome side effects with 2 capsules, there was a possibility to reduce the dose again. Furthermore, all patients were asked whether they estimated their vigilance impairment to be ameliorated by treatment.

After 6 treatment weeks, all examinations, including questionnaires, actigraphy, conventional nocturnal polysomnography, MWTs, and psychomotor vigilance tests, were repeated. Primary outcome measures included improvements in FSS and ESS values, and in mean sleep latencies on MWT.

Standard protocol approvals, registrations, and patient consents. The study was approved by the local ethical standards committee on human experimentation (Ethikkommission des Kantons Zurich) and by Swissmedic, the Swiss agency for therapeutic products. All study participants gave oral and written consent to all study procedures.

Statistics. Statistical analyses were performed by using correlation analyses (Pearson and Spearman), Student $t$ tests, Mann-Whitney $U$ tests, and multivariate regression analyses. Parametric tests were 2-tailed.

RESULTS Demographic data and baseline characteristics of both the treatment and the placebo group can be found in table 1. Patients in both groups did not differ in age, sex distribution, severity of TBI, or duration since trauma. At study inclusion, the duration since trauma was 1 to 3 years in all patients. After baseline examinations, we excluded 4 patients because of obstructive sleep apnea and 1 patient be- 


\begin{tabular}{|c|c|c|c|c|}
\hline \multirow[t]{2}{*}{ Table 1} & \multicolumn{4}{|c|}{$\begin{array}{l}\text { Baseline characteristics and changes from baseline in } 20 \text { patients } \\
\text { with traumatic brain injury, } 10 \text { treated with placebo and } 10 \\
\text { treated with modafinil }\end{array}$} \\
\hline & & Placebo & Modafinil & $p$ Value \\
\hline \multicolumn{5}{|c|}{ General findings } \\
\hline \multicolumn{2}{|l|}{ Age, $y$} & $43 \pm 19$ & $37 \pm 9$ & \\
\hline \multicolumn{2}{|c|}{ Sex, \% male } & 90 & 80 & \\
\hline \multicolumn{2}{|c|}{ Glasgow Coma Scale } & $8 \pm 4$ & $7 \pm 4$ & \\
\hline \multicolumn{2}{|c|}{ Pathologic CT findings, \% } & 60 & 50 & \\
\hline \multicolumn{2}{|c|}{ Interval after trauma, y } & $2.0 \pm 1.2$ & $1.8 \pm 0.9$ & \\
\hline \multicolumn{2}{|c|}{ High dosage (modafinil group: 200 mg), \% } & 80 & 80 & \\
\hline \multicolumn{2}{|c|}{ Side effects, \% } & 10 & 20 & \\
\hline \multicolumn{5}{|c|}{ Baseline findings } \\
\hline \multicolumn{2}{|l|}{ ESS } & $8.2 \pm 3.7$ & $10.0 \pm 4.2$ & \\
\hline \multicolumn{2}{|l|}{ FSS } & $5.0 \pm 1.4$ & $4.6 \pm 0.8$ & \\
\hline \multicolumn{2}{|c|}{ Beck Depression Inventory } & $11 \pm 9$ & $9 \pm 6$ & \\
\hline \multicolumn{2}{|c|}{ Beck Anxiety Inventory } & $10 \pm 11$ & $10 \pm 9$ & \\
\hline \multicolumn{2}{|c|}{ PSG: sleep efficiency, $\%$} & $87 \pm 5$ & $88 \pm 11$ & \\
\hline \multicolumn{2}{|c|}{ PSG: sleep latency to NREM2, $\min$} & $20 \pm 16$ & $16 \pm 18$ & \\
\hline \multicolumn{2}{|c|}{ PSG: apnea-hypopnea index } & $4.3 \pm 3.8$ & $2.6 \pm 3.3$ & \\
\hline \multicolumn{2}{|c|}{ PSG: periodic limb movements index } & $0.8 \pm 0.9$ & $2.1 \pm 4.4$ & \\
\hline \multicolumn{2}{|c|}{ MWT: mean sleep latency, min } & $18.7 \pm 9.3$ & $15.6 \pm 11.4$ & \\
\hline \multicolumn{2}{|c|}{ Actigraphy: awake per $24 \mathrm{~h}, \%$} & $39 \pm 13$ & $41 \pm 11$ & \\
\hline \multicolumn{2}{|c|}{ Actigraphy: sleep elongation weekend, $\mathrm{h}$} & $0.5 \pm 0.7$ & $0.8 \pm 1.0$ & \\
\hline \multicolumn{2}{|c|}{ PVT: velocity, s } & $3.7 \pm 0.6$ & $3.8 \pm 0.5$ & \\
\hline \multicolumn{2}{|c|}{ PVT: no. of lapses } & $3.7 \pm 4.3$ & $2.8 \pm 1.9$ & \\
\hline \multicolumn{5}{|c|}{ Changes from baseline } \\
\hline \multicolumn{2}{|l|}{ ESS } & $0.7 \pm 1.8$ & $-2.3 \pm 2.3$ & $0.005^{b}$ \\
\hline \multicolumn{2}{|l|}{ FSS } & $0.0 \pm 0.6$ & $-0.8 \pm 1.0$ & $0.07^{b}$ \\
\hline \multicolumn{2}{|c|}{ Beck Depression Inventory } & $-0.4 \pm 3.5$ & $1.1 \pm 3.2$ & NS \\
\hline \multicolumn{2}{|c|}{ Beck Anxiety Inventory } & $0.5 \pm 5.2$ & $-0.1 \pm 1.8$ & NS \\
\hline PSG: sle & efficiency, \% & $0.8 \pm 5.7$ & $0.0 \pm 5.1$ & NS \\
\hline PSG: sle & latency to NREM2, min & $-13.6 \pm 16.4$ & $2.7 \pm 14.7$ & $0.03^{b}$ \\
\hline MWT: $m$ & n sleep latency, $\min$ & $0.4 \pm 6.2$ & $8.4 \pm 9.6$ & $0.04^{\mathrm{b}}$ \\
\hline Actigra & : awake per $24 \mathrm{~h}, \%$ & $-0.3 \pm 4.7$ & $1.9 \pm 3.8$ & NS \\
\hline PVT: ve & ity, s & $0.0 \pm 0.2$ & $-0.2 \pm 0.4$ & NS \\
\hline PVT: no & lapses & $0.8 \pm 2.9$ & $1.3 \pm 2.6$ & NS \\
\hline
\end{tabular}

Abbreviations: ESS = Epworth Sleepiness Scale; FSS = Fatigue Severity Scale; MWT = maintenance of wakefulness test; NS = not significant; PSG = polysomnography; PVT = psychomotor vigilance test.

a Mean values are given with SDs.

${ }^{b}$ Mann-Whitney U test.
EDS. Again, ESS values at baseline did not differ between the 2 groups (modafinil: $8.2 \pm 3.7$, placebo: $10.0 \pm 4.2$ ). Relevant sleep-related breathing or movement disorders were not present in either group. The respective measures such as the apneahypopnea index or the periodic limb movement index were similar in the 2 groups. Also, actigraphy measures did not differ between groups. Beck Depression and Anxiety Inventories revealed similar results in the 2 groups. In both treatment groups, the dose of study medication was doubled in 8 patients. It was not necessary in any of these patients to reduce the dose again because of side effects. None of our patients discontinued treatment.

After 6 weeks of treatment, the decrease in FSS scores was higher in the modafinil group $(-0.8 \pm$ 1.0) than in the placebo group $(0.0 \pm 0.6)$, but this finding was not significant after correction for the independent variables sex, age, TBI severity, and Beck Depression and Anxiety scale values (figure 2). Fatigue was still present in all 8 patients in the placebo group and in 4 patients in the modafinil group. When selecting only patients with fatigue (FSS $\geq 4.0)$ at baseline, decreases in FSS scores were not higher in the modafinil group.

After 6 treatment weeks and compared with the placebo group, the decrease in ESS scores was higher in the modafinil group $(-2.3 \pm 2.3$ compared with $0.7 \pm 1.8, p=0.005$; figure 2 ). This finding remained significant after correction for the same independent variables, and also when selecting only those patients with EDS (ESS $\geq 10$ ) at baseline. Under treatment, EDS was still present in 3 patients in each group.

The objective measurement of the ability to remain awake at daytime under nonstimulating conditions revealed a significant increase compared with baseline of the mean sleep latency on MWT in the treatment group (8.4 \pm 9.6 minutes) compared with the placebo group ( $0.4 \pm 6.2$ minutes) ( $p=0.04$; figure 2). Patients under placebo treatment fell asleep quicker at nocturnal polysomnography than at baseline $(-13.6 \pm 16.4$ minutes $)$ after 6 treatment weeks, which probably reflects habituation to the sleep laboratory environment. Patients treated with modafinil, however, had no change in sleep latencies compared with baseline ( $2.7 \pm 14.7$ minutes), which again indicates decreased sleep pressure compared with the placebo group ( $p=0.03$ ). Conversely, the psychomotor vigilance test did not reveal differences between the groups: both reaction times and number of lapses were similar. Actigraphy revealed an increase of time awake per 24 hours in the modafinil group by 1.9 hours, whereas time awake decreased mildly $(-0.3$ hours) in the placebo group ( $p=$

0.33). cause of chronic sleep deprivation. A flow diagram depicting the passage of participants through this study is given in figure 1 .

At baseline, 8 patients in every group were diagnosed with fatigue. FSS values at baseline did not differ between the 2 groups: mean FSS values were $5.0 \pm 1.4$ in the modafinil group and $4.6 \pm 0.8$ in the placebo group. Five patients in the placebo group and 6 in the modafinil group were diagnosed with 


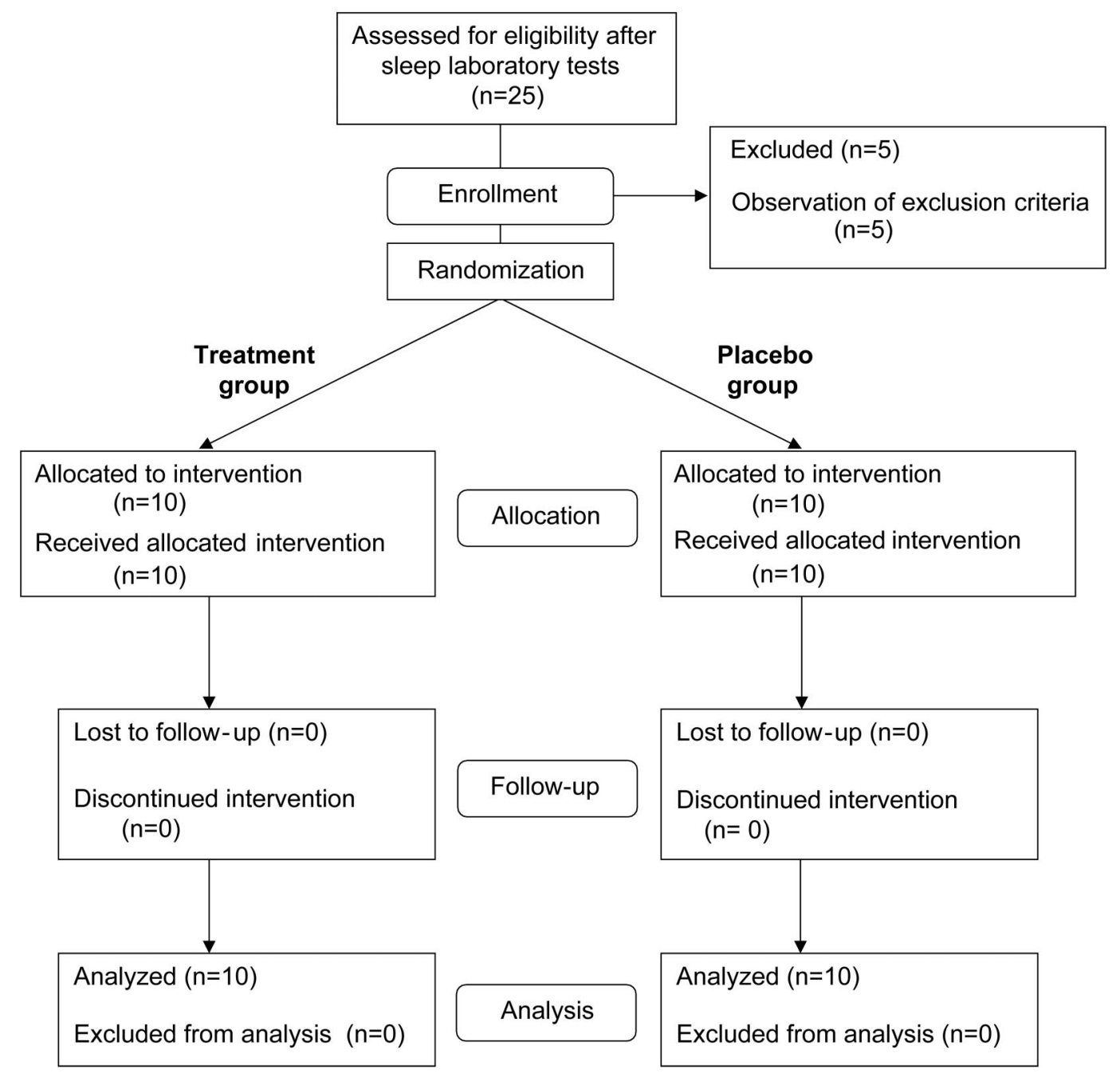

The overall subjective estimation of patients on vigilance impairment amelioration was similar in the 2 groups (table 2). These subjective self-estimations did not correlate with the FSS, the ESS, or MWT results. Depression and anxiety scale values did not change from baseline in either group. Thus, treatment-although improving objective measures of sleepiness-had no impact on quality of life. This may not be surprising, because 2.3 points on the ESS is not clinically significant, although 8.4 minutes on the MWT might be.

Side effects were similar in the 2 groups. One placebo patient reported a reduction in appetite, 1 modafinil patient reported nausea and mild and transient stomachache, and 1 modafinil patient reported mild arthralgia in the shoulders.

DISCUSSION The main outcome of this first double-blind, randomized, placebo-controlled study that included electrophysiologic sleep laboratory tests in 20 patients with TBI indicates that 100 to $200 \mathrm{mg}$ modafinil is effective and well tolerated in the treatment of posttraumatic EDS but not of fatigue. ESS values decreased by 2.3 points and mean sleep latencies on MWT increased by 8.4 minutes under treatment. Furthermore, patients with TBI who were treated with modafinil stayed awake 1.9 hours longer per day, whereas placebo-treated patients did not reveal an increase of their time awake. This potentially important difference, however, remained nonsignificant in this small trial.

Several studies observed an improvement of EDS with modafinil in a variety of disorders, including narcolepsy, obstructive sleep apnea, and chronic shift work. ${ }^{16-18}$ After TBI, EDS and fatigue are frequent complications. ${ }^{4}$ Earlier studies showed a potential benefit of modafinil for the treatment of posttraumatic vigilance impairment. ${ }^{19,20}$ Under treatment with 100 to $400 \mathrm{mg}$ modafinil (once every morning), Teitelman reported increased wakefulness and attention of posttraumatic hypersomnia in 10 patients who had closed TBI. ${ }^{19}$ Unfortunately, objective mea- 
Figure 2 Changes from baseline in ESS and FSS values, and in mean sleep latencies on MWT (in minutes)

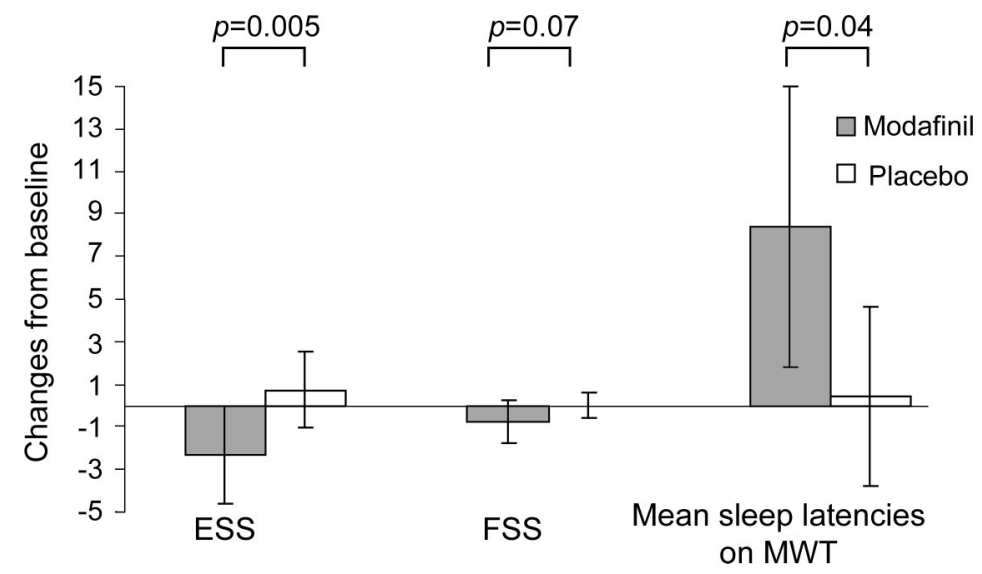

ESS $=$ Epworth Sleepiness Scale; FSS $=$ Fatigue Severity Scale; MWT = maintenance of wakefulness test. ceived placebo. This may have 2 reasons: First, based on findings in our earlier studies and in accordance with other authors, we believe that many patients with TBI tend to underestimate their sleep problems. ${ }^{4,21}$ In our first study on posttraumatic sleepwake disturbances, we found very low mean sleep latencies on multiple sleep latency tests in patients with TBI who reported that they would not be sleepy at daytime. ${ }^{4}$ Likewise, in the present study, the effect of modafinil on ESS values is smaller than in trials examining patients with narcolepsy or sleep apnea, whereas the improvement on the MWT is similar to the findings in these disorders. ${ }^{16,17}$ Therefore, the question whether patients with TBI develop a specific type of anosognosia toward sleep-wake disturbances remains to be elucidated in systematic studies. Second, in this pilot study, we administered only 100 to $200 \mathrm{mg}$ modafinil. We assume that higher doses might have a higher and clinically more significant impact on vigilance.

The lacking effect of modafinil on fatigue is remarkable. Recently, a similar outcome was observed in patients with Parkinson disease, with an improvement of EDS but not of fatigue with modafinil treatment. ${ }^{22}$ In fatigue related to multiple sclerosis or diencephalic stroke, however, modafinil was effective. ${ }^{23}$ In general, fatigue is an ill-defined symptom that probably is influenced by several factors, including physical exhaustibility, sleep-wake disturbances, and disorders of mood. Furthermore, it is not clear whether fatigue and EDS belong to the same clinical spectrum. Our result of effectiveness of modafinil for EDS but not fatigue indicates that the 2 symptoms are distinct entities, probably with different pathophysiologic pathways.

trial. In our trial as much as in this cited study, however, there is the possibility of a ceiling effect, where no higher dose of modafinil would further improve vigilance in our patients. However, because we only administered 100 to $200 \mathrm{mg}$, we cannot rule out that higher doses might add benefit for our patients.

In our study, the subjective estimation of treated patients regarding an improvement of vigilance during medication was not different from those who re-

\begin{tabular}{|llll|}
\hline Table 2 & $\begin{array}{l}\text { Overall subjective estimation } \\
\text { of vigilance impairment } \\
\text { (fatigue, excessive daytime } \\
\text { sleepiness) amelioration }\end{array}$ & \\
Much better & 1 & 0 & Placebo \\
Better & 1 & 3 & 0.23 \\
Somewhat better & 1 & 3 & \\
Unchanged & 7 & 3 & \\
Worse & 0 & 1 & \\
\hline
\end{tabular}

\section{AUTHOR CONTRIBUTIONS}

Statistical analyses were performed by Christian R. Baumann.

\section{DISCLOSURE}

Dr. Kaiser, Dr. Valko, Dr. Werth, Ms. Thomann, and Ms. Meier report no disclosures. Prof. Stocker has served on scientific advisory boards for KCI Europe Holding BV and Baxter International Inc.; has received funding for travel or speaker honoraria from Fresenius Medical Care and Bio-Synthesis Inc.; and receives research support from the Swiss National Foundation, SUVA Foundation, the Swiss Academy for Medical Sciences, and Swisstransplant. Prof. Bassetti serves as Chief Editor of the Swiss Archives of Neurology and Psychiatry and on the editorial boards of the Journal of Sleep Research, Sleep Medicine, Somnology, and the Belgian Neurological Journal; has served on scientific advisory boards for and received speaker honoraria from Boehringer Ingelheim, Lundbeck Inc., Novartis, Pfizer Inc, ResMed Inc., and UCB; receives research support from Lundbeck Inc., Respironics, Inc., ResMed Inc., Vifor Pharma, and UCB; and receives institutional research support from the Zurich Center for Integrative Human Physiology and the Swiss National Science Foundation. Dr. Baumann has served on scientific advisory boards for and received speaker honoraria from Boehringer Ingelheim, UCB, and GlaxoSmithKline; receives research support from UCB, Cephalon, and Novartis; and receives institutional research support from the Swiss National Foundation. 

2005;6:507-513.

\section{REFERENCES}

1. National Center for Injury Prevention and Control. Traumatic Brain Injury in the United States: Emergency Department Visits, Hospitalizations, and Deaths. Atlanta, GA: Centers for Disease Control and Prevention; 2006.

2. Zaloshnja E, Miller T, Janglois JA, Selassie AW. Prevalence of long-term disability from traumatic brain injury in the civilian population of the United States. J Head Trauma Rehabil 2008;23:394-400.

3. Murray CJL, Lopez AD. Global mortality, disability and the contribution of risk factors: Global Burden of Disease Study. Lancet 1997;349:1436-1443.

4. Baumann CR, Werth E, Stocker R, Ludwig S, Bassetti CL. Sleep-wake disturbances 6 months after traumatic brain injury: a prospective study. Brain 2007;130:1873-1883.

5. Masel BE, Scheibel RS, Kimbark T, Kuna ST. Excessive daytime sleepiness in adults with brain injuries. Arch Phys Med Rehabil 2001;82:1526-1532.

6. Rao V, Spiro J, Vaishnavi S, et al. Prevalence and types of sleep disturbances acutely after traumatic brain injury. Brain Inj 2008;22:381-386.

7. Castriotta RJ, Wilde MC, Lai JM, Atanasov S, Masel BE, Kuna ST. Prevalence and consequences of sleep disorders in traumatic brain injury. J Clin Sleep Med 2007;3:349-356.

8. Baumann CR, Bassetti CL, Valko PO, et al. Loss of hypocretin (orexin) neurons with traumatic brain injury. Ann Neurol 2009;66:555-559.

9. Bloch KE, Schoch OD, Zhang JN, Russi EW. German version of the Epworth Sleepiness Scale. Respiration 1999; 66:440-447.

10. Valko PO, Bassetti CL, Bloch KE, Held U, Baumann CR. Validation of the fatigue severity scale in a Swiss cohort. Sleep 2008;31:1601-1607.

11. Homaifar BY, Brenner LA, Gutierrez PM, et al. Sensitivity and specificity of the Beck Depression Inventory-II in persons with traumatic brain injury. Arch Phys Med Rehabil 2009;90:652-656.

12. King MA, Jaffre MO, Morrish E, Shneerson JM, Smith IE. The validation of a new actigraphy system for the mea-
13. Rechtschaffen A, Kales A. A Manual of Standardized Terminology, Techniques and Scoring System for Sleep Stages of Human Subjects. Washington, DC: US Public Health Service, US Government Printing Office; 1968.

14. Littner MR, Kushida C, Wise M, et al; Standards of Practice Committee of the American Academy of Sleep Medicine. Practice parameters for clinical use of the multiple sleep latency test and the maintenance of wakefulness test. Sleep 2005;28:113-121.

15. Dorrian J, Lamond N, Holmes AL, et al. The ability to self-monitor performance during a week of simulated night shifts. Sleep 2003;26:871-877.

16. Broughton RJ, Fleming JA, George CF, et al. Randomized, double-blind, placebo-controlled crossover trial of modafinil in the treatment of excessive daytime sleepiness in narcolepsy. Neurology 1997;49:444-451.

17. Black JE, Hirshkowitz M. Modafinil for treatment of residual excessive sleepiness in nasal continuous positive airway pressure-treated obstructive sleep apnea/hypopnea syndrome. Sleep 2005;28:464-471.

18. Czeisler CA, Walsh JK, Roth T, et al; U.S. Modafinil in Shift Work Sleep Disorder Study Group. Modafinil for excessive sleepiness associated with shift-work sleep disorder. N Engl J Med 2005;353:476-486.

19. Teitelman E. Off-label uses of modafinil. Am J Psychiatry 2001;158:1341.

20. Jha A, Weintraub A, Allshouse A, et al. A randomized trial of modafinil for the treatment of fatigue and excessive daytime sleepiness in individuals with chronic traumatic brain injury. J Head Trauma Rehabil 2008;23:52-63.

21. Chaumet G, Quera-Salva MA, Macleod A, et al. Is there a link between alertness and fatigue in patients with traumatic brain injury? Neurology 2008;71:1609-1613.

22. Tyne HL, Taylor J, Baker GA, Steiger MJ. Modafinil for Parkinson's disease fatigue. J Neurol 2010;257:452-456.

23. Brioschi A, Gramigna S, Werth E, et al. Effect of modafinil on subjective fatigue in multiple sclerosis and stroke patients. Eur Neurol 2009;62:243-249.

\section{Get the Latest Drug Recalls and Warnings. Give the Best Patient Care}

The American Academy of Neurology and the Health Care Notification Network have teamed up to offer AAN members a FREE online service that delivers timely neurology-specific FDAmandated patient safety drug alerts electronically.

Don't miss this opportunity to provide the best—and safest—possible care for your patients: www.aan.com/view/FDAalert 